\title{
Tendances vers l'académisation de la formation postgrade en psychothérapie
}

\section{Peter Schulthess}

La tendance à l'académisation de la formation postgrade en psychothérapie est évidente. Pendant longtemps, les universités se sont vues comme des lieux d'enseignement scientifique et non de formation professionnelle. La formation psychothérapeutique en tant que formation professionnelle s'est déroulée en parallèle ou par la suite dans des instituts de formation privés. Cela a également beaucoup changé en Suisse. Pratiquement toutes les universités offrent aujourd'hui leurs propres cursus (filières) de formation postgrade en psychothérapie, principalement en thérapie comportementale. Cependant, d'autres directions peuvent également être trouvées, selon les préférences des titulaires (h/f) des chaires respectives. En règle générale, ces cursus (filières) sont réalisés en partenariat avec un institut privé existant.

Les avantages sont évidents : Lien avec l'enseignement théorique de la recherche et de la thérapie scientifique, diplôme universitaire (MAS) avec une qualification académique supplémentaire après la maîtrise ou le doctorat.

Qu'est-ce que cela signifie pour les formations postgrade privées en Suisse qui ne sont pas affiliées à une université ? La pression exercée au cours des procédures d'accréditation après l'intensification de la recherche et de l'évaluation scientifique est indubitable et a souvent conduit aux charges respectives. Cependant, la recherche ne peut se faire que dans un réseau plus vaste, au moins la recherche empirique. Cette dernière est obligatoire aussi longtemps que le principe de la médecine fondée sur les preuves selon le modèle médical de la médecine « evidence based medicine "s'applique également en psychothérapie afin de rester reconnu comme une procédure. Comment les institutions privées peuvent-elles se positionner à cet égard ? Ont-elles un désavantage concurrentiel à long terme ? Quelles autres formes de coopération que le partenariat dans le cadre d'une filière de formation postgrade du MAS sont envisageables pour accéder à des fonds et à un savoir-faire de recherche, ainsi que pour transmettre des connaissances de base basées sur la preuve dans le domaine en plein essor de la psychothérapie ? Tôt ou tard, la diversité de la psychothérapie en Suisse sera-t-elle menacée? $\mathrm{Au}$ cours de la première ronde d'accréditation, certaines orientations thérapeutiques ont déjà été éliminées, y compris celles qui sont reconnues scientifiquement dans d'autres pays.
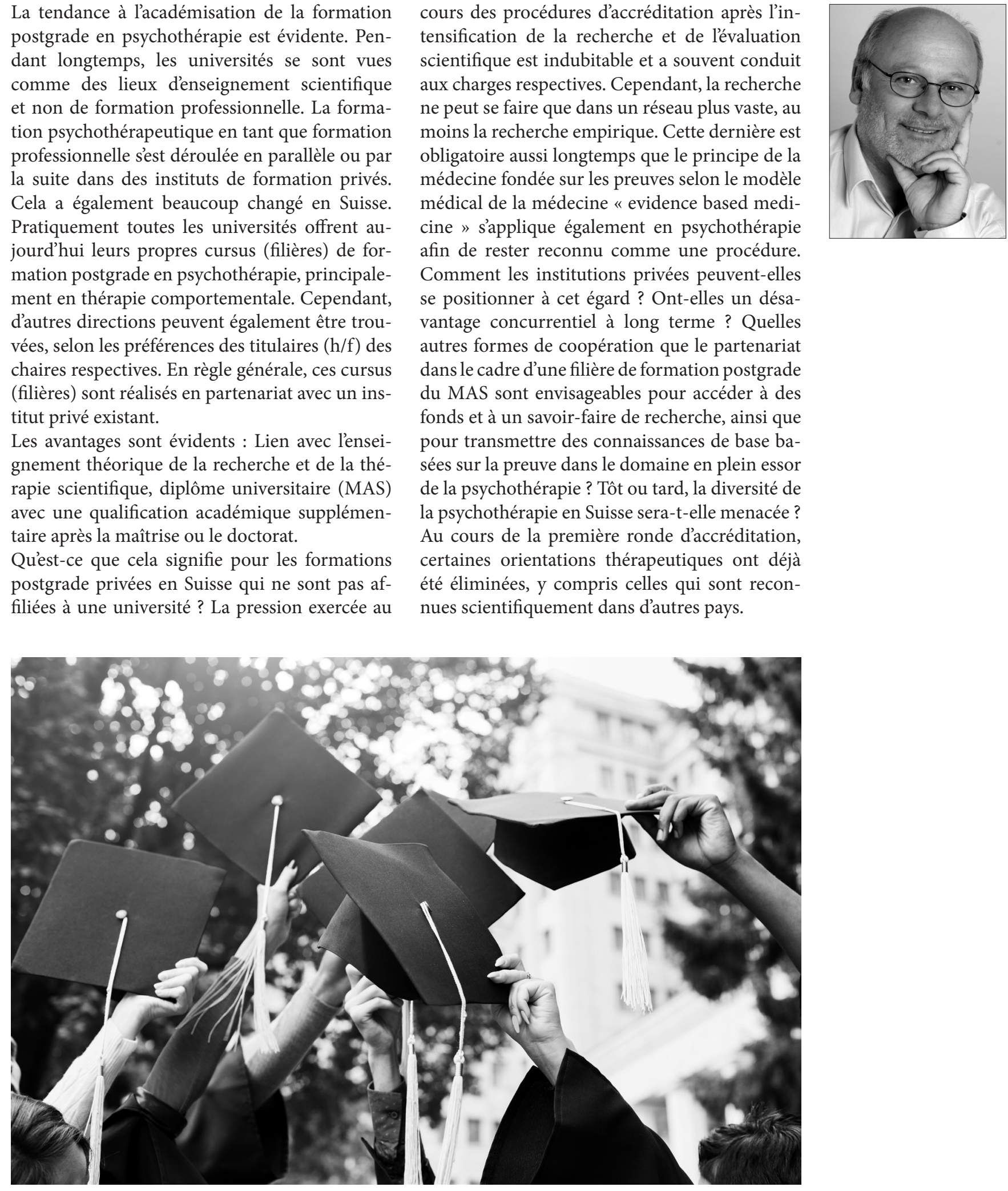
La Loi sur les professions de la psychologie (LPsy) a consolidé la distinction entre la formation ou étude de base (étude de la psychologie) et la formation continue. Les chevauchements entre l'étude de la psychologie et la formation continue en psychothérapie sont interdits et ne doivent pas avoir lieu, même si cela doit être considéré comme incompréhensible et injustifié dans des cas individuels. Cette séparation stricte contraste avec une tendance à la hausse dans d'autres pays européens. Depuis des années, l'Université privée Sigmund Freud de Vienne (SFU) et plusieurs universités sœurs d'autres pays proposent un cursus de psychothérapie scientifique qui combine les connaissances scientifiques de base et la formation thérapeutique avec la pratique clinique, le tout dans le sens d'une formation professionnelle scientifique en tant que psychothérapeute. En Allemagne, une révision de la Loi sur la psychothérapie a récemment été adoptée, qui prévoit des cours directs en psychothérapie. La séparation entre les études de base en psychologie et la formation continue en psychothérapie a donc ainsi été abolie, précisément en Allemagne qui est le modèle qui a inspiré la Suisse dans sa LPsy. À l'avenir, il sera possible détudier la psychothérapie directement à partir du baccalauréat, ce qui implique également un droit à la pratique de la profession avec le diplôme. Combien de temps faudra-t-il pour que la LPsy soit révisée en conséquence afin de pouvoir proposer de tels cours dans les universités suisses? Qu'est-ce que cela signifie pour les instituts privés?

Eh bien, la Suisse met généralement une dizaine d'années à suivre le modèle allemand. Néanmoins, la discussion devrait avoir lieu aujourd'hui. Quels sont les avantages d'un tel programme d'études direct ? Quels sont ses inconvénients ? Dans quelle mesure cela met-il en danger la diversité des disciplines psychothérapeutiques? Quels types de coopérations entre instituts privés et universités sont envisageables ? Quelles sont vos premières expériences?
Afin d'approfondir ces questions, l'ASP organise une conférence le 28 mars sur le thème "Tendances vers l'académisation de la formation continue en psychothérapie ».

Deux modèles de partenariats existants entre des instituts privés et des universités sont présentés, qui proposent depuis plusieurs années une filière de formation postgrade accréditée avec diplôme MAS : "Psychothérapie systémique comportementale " à la ZHAW et "Psychothérapie centrée sur la personne » à l'Université de Bâle. Imke Knafla et Jens Gaab, et les instituts partenaires privés Rainer Bürki et Julia Besewski, donneront des informations sur les raisons pour lesquelles ils se sont engagés dans cette voie et quels sont, de leur point de vue et selon leur expérience, les avantages, mais aussi les problèmes possibles. La professeure Jutta Fiegl, responsable du cours détudes psychothérapeutiques à la SFU, rendra compte de la coopération avec des instituts privés dans le cadre de ce cours d'études direct et des expériences qui en ont été faites. Nikolaus Melkop, membre du comité de la Chambre fédérale des psychothérapeutes (h/f) d'Allemagne, nous informe sur la réforme de la Loi sur les psychothérapeutes et sur ce que l'on peut attendre ou craindre des études directes. Le professeur Christoph Flückiger commentera le sujet du point de vue de l'Université de Zurich.

Ne manquez pas l'occasion d'obtenir de l'information de première main sur ces sujets et de participer au discours !

\section{Peter Schulthess est membre du comité de l'ASP.}

Conférence de I'ASP à Zurich, samedi 28 mars 2020

Sujet : «Tendances vers l'académisation de la formation postgrade en psychothérapie. »

Intervenants: Imke Knafla (ZHAW), Jens Gaab (Université de Bâle), Jutta Fiegl (SFU Vienne), Christoph Flückiger (Université de Zurich), Nikolaus Melkop (Chambre fédérale des psychothérapeutes), Rainer Bürki (CPA), Julia Besewski (ZSB) 